\title{
Hemomediastinum and bilateral hemothorax with extensive angiomatosis of anterior mediastinum
}

\author{
Sh. Karimi1 , F. Mohammadi1, S. Pejhan², M. Bakhshayeshkaram³, \\ P. Akhavan-Azari1, M. Bahadori1
}

ABSTRACT: Hemomediastinum and bilateral hemothorax with extensive angiomatosis of anterior mediastinum. Sh. Karimi, F. Mohammadi, S. Pejhan, M. Bakhshayeshkaram, P. Akhavan-Azari, M. Bahadori.

Angiomatosis is a rare, benign but clinically extensive and serious vascular lesion of soft tissue. Hereby, we report a case of diffuse angiomatosis of mediastinum, presenting with hemomediastinum and bilateral massive hemothorax in a 19-year old boy.

On medical imaging, mediastinal widening along with enhanced small vessels and capillaries were detected. On operation, hemorrhagic sponge-like vascular tissue patches were seen extensively in pericardium, pleura, lymph node and thymus as well. The lesion was debulked.

The pathologic evaluation revealed characteristic features of soft tissue angiomatosis involving thymus, lymph node, pleura, pericardium and its fibrofatty tissue.

Diffuse angiomatosis should be considered for differential diagnosis of vascular lesions of anterior mediastinum. Monaldi Arch Chest Dis 2006; 65: 3, 172-174.

Keywords: Angiomatosis, mediastinum, thymus.

1 Department of pathology, NRITLD, SBU,

2 Department of thoracic surgery, $S B U$,

3 Department of radiology, SBU, Tehran, Iran.

Correpondence: Shirin Karimi, Department of Pathology, National Institute of Tuberculosis and Lung Disease (NRITLD), Shahid Beheshti University of Medical Science (SBUMS), Tehran, Iran; e-mail: shirinkarimi_1@yahoo.com

Angiomatosis is a rare benign vascular lesion representing almost $4 \%$ of vascular tumors in children and adolescents. These lesions occur in soft tissue and are clinically extensive covering large parts of the body in a continuous pattern [1]. A diffuse angiomatosis affecting thoracal organs is a rare, frequently fatal disorder occurring mainly in childhood [2]. Hereby, we present a 19-year old boy with diffuse mediastinal angiomatosis, presenting with bilateral hemothorax and hemomediastinum.

\section{Case report}

A 19-year old boy was referred to Masih Daneshvari hospital due to mediastinal widening and bilateral hemothorax. The patient had a history of cough, exertional dyspnea, fever and weight loss (5 kg) from 1.5 months ago. He had also history of bronchiectasis from infancy. Chest x-ray, chest Computed Tomography (CT)-scan with contrast and finally spiral CT-scan were performed. The medical imaging revealed dense fluid collections and enhanced tubular structures within all mediastinal compartments, bilateral pleural effusion, and passive atelectasis of left lower lobe (fig. 1). On admission the patient's condition was critical having dyspnea at rest, pallor, tachycardia and hypotension with hemoglobin about $4 \mathrm{mg} / \mathrm{dl}$. After blood transfusion due to severe anemia, bilateral tube thoracostomy was performed and $1200 \mathrm{ml}$ of blood was drained from both hemithoraces. Rigid bronchoscopy revealed no endobronchial or any source of hemorrhage in trachea and main bronchus.

A full median sternotomy was performed. Hemorrhagic sponge-like vascular tissue patches similar to cavernous hemangioma were found all over the pericardium, mediastinum, and right pleura (fig. 2). Hemopericardium was also found in addition of 2-3 fenestrations on pericardium. Root of aorta and soft tissue around the innominate vessels were also involved but phrenic nerves were spared. The lesion was debulked as much as possible.

The frozen sections revealed numerous vascular channels of variable sizes with prominent thick walls embedded in fibro-fatty tissue associated with bloody lakes and infiltration of hemosiderin laden macrophages. The differential diagnosis was between arterious-venous malformation and hamartomatous vascular lesions. Microscopic examination of permanent sections from thymus, pericardium, lymph nodes, pleura and surrounding fibro-fatty tissue (fig. 3) revealed diffuse angiomatosis associated with extensive areas of hemorrhage. The vascular channels were composed of variable size veins, arteries, capillaries and focal cavernous vascular channels as well. These vessels 


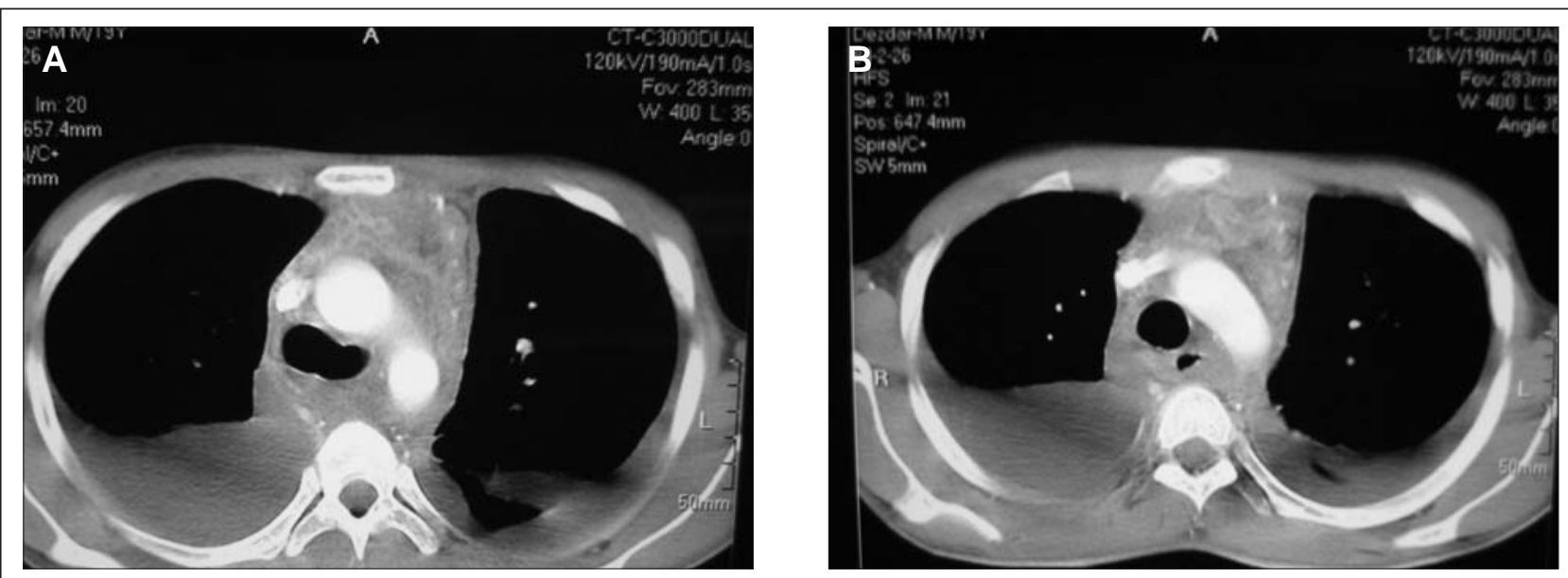

Figure 1. - CT scan of the thorax showing dense fluid collections, bilateral pleural effusion, and passive atelectasis of left lower lobe.
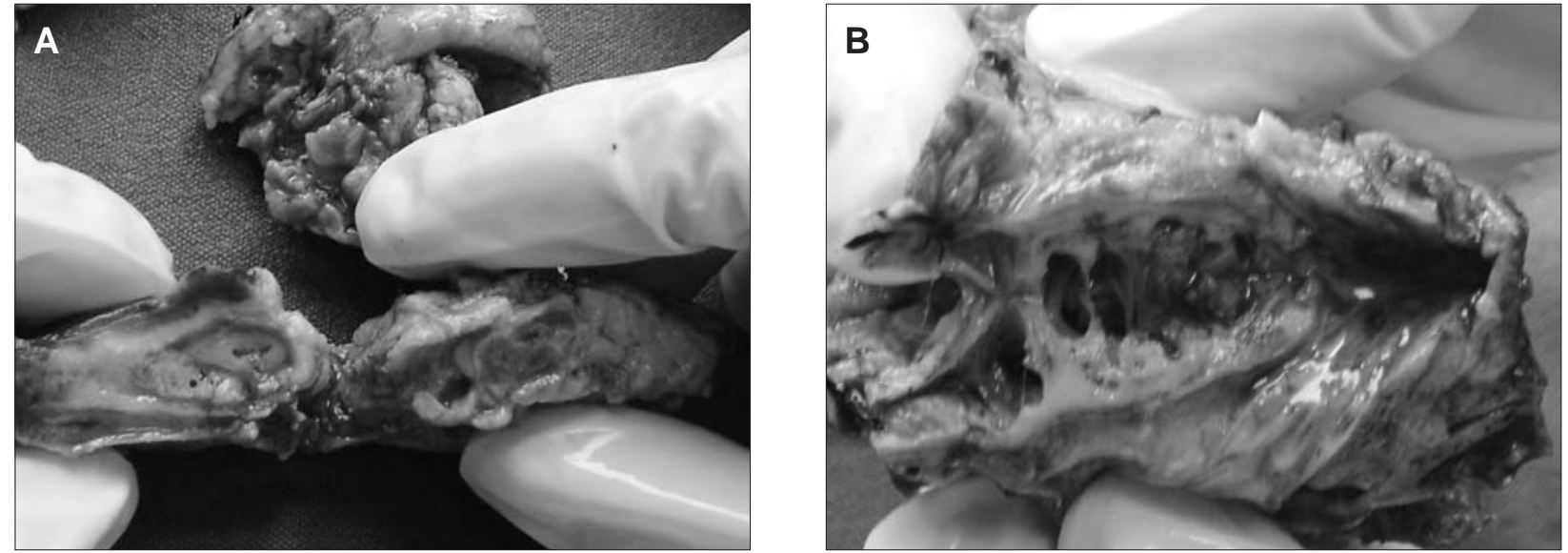

Figure 2. - Macroscopic appearance of the removed angiomatosis: hemorrhagic sponge-like vascular tissue patches similar to cavernous hemangioma.

were scattered haphazardly through out these tissues. The venous channels were irregular and herniated with a thick wall showing many small vessels in it or just adjacent to it. All vascular channels were lined by flat endothelium and there were scattered nerve bundles among them. A few thyroid follicles were embedded in fibrofatty tissue and lymph nodes of anterior mediastinum.

After surgery, the patient's clinical condition improved significantly. During the 6 month follow-up, clinical condition of the patient was good and his last chest x-ray was normal. Thyroid scan was performed as well which was normal too. The patient is still under observation.

\section{Discussion}

This case is particularly interesting because of simultaneous presentation of bilateral hemothorax and mediastinal widening due to hemomediastinum. The impression of surgeons and radiologists were mostly in favor of hemangioma of mediastinum. Characteristic diffuse multiple tissue involvement and microscopic pathological findings revealed the diagnosis of diffuse angiomatosis. This lesion is rarely reported specially in the differential diagnosis of hemothorax and hemomediastinum.
To the best of our knowledge, there are a few reports of diffuse angiomatosis of mediastinum. It had never been reported occurring in mediastinum until 1995 [4]. The authors report an original case of angiomatosis located in the anterosuperior part of mediastinum in a 17-year old girl [1]. A case of angiomatosis in the neck, larynx and mediastinum was also reported [5] and a 37-year old patient with extensive diffuse angiomatosis affecting mediastinum, pericardium and pleura was described as well [2].

Histologically angiomatosis may be presented by one of the following patterns: The more common pattern consists of a mélange of large, venous, cavernous, and capillary size vessels scattered haphazardly throughout the soft tissue [3]. The second pattern which occurs in a small number of cases is virtually identical to that of capillary hemangioma, except that the nodules of tumor diffusely infiltrate the surrounding soft tissue. Diagnosis of these unusual tumors may be difficult. The distinction between angiomatosis and intramuscular hemangioma is fundamentally based on clinical rather than pathological criteria. However, we believe that the irregular venous channels with clustered small vessels in their walls are characteristic of angiomatosis. Angiomatosis is a poorly understood entity belonging to the vascular tumors family [4]. Because of the prominent amount of fat 

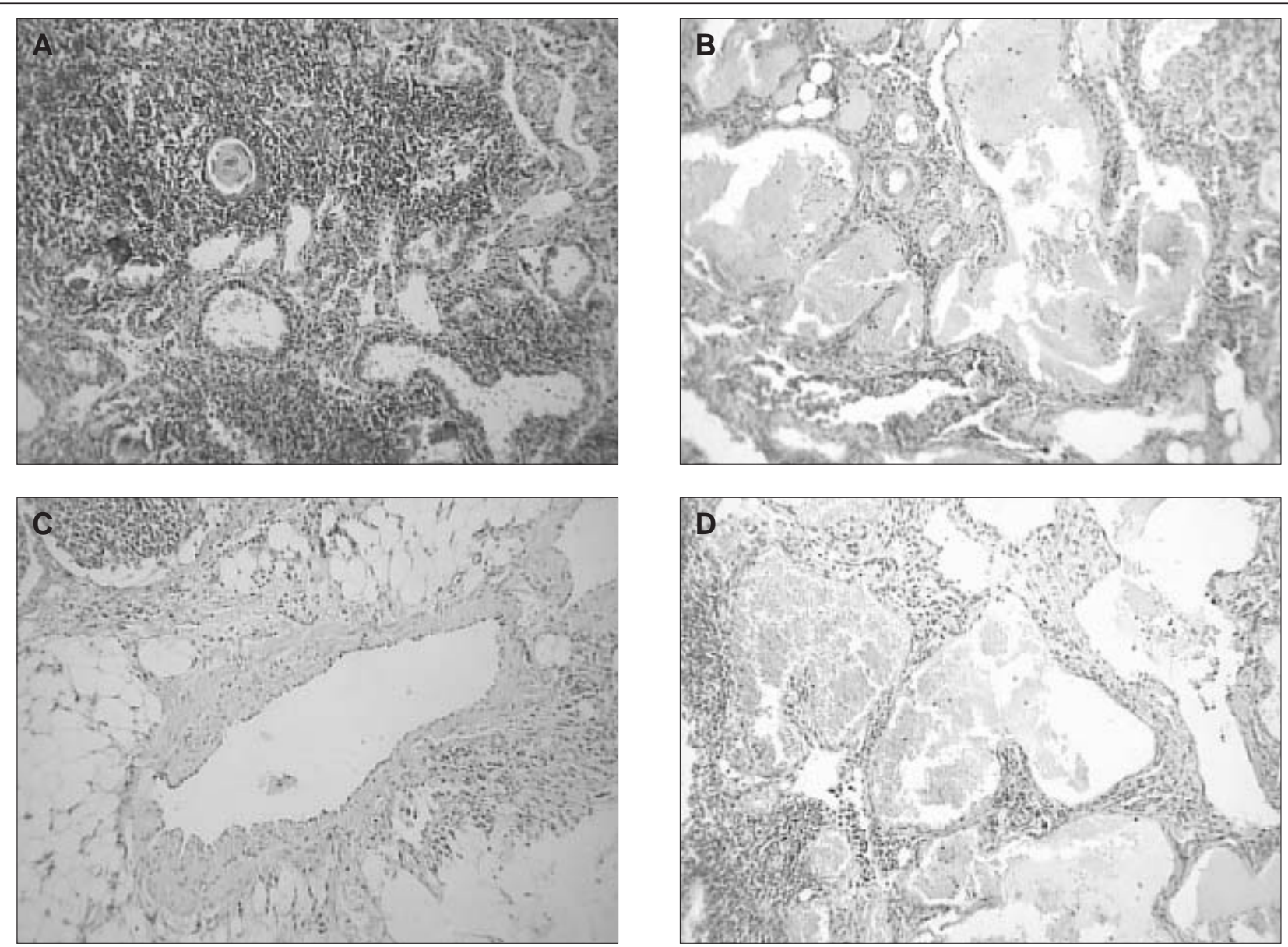

Figure 3. - Microscopic appearance of the angiomatosis in histologic sections from different involved tissues.

present in these lesions, previous authors suggest that angiomatosis is probably best regarded as a more generalized mesenchymal proliferation [3]. It may also represent a multiple low-flow malformation [2]. Presence of ectopic thyroid tissue and nerve bundles in our case presentation may also suggest a hamartomatous origin for this lesion. Additional studies based on cytogenetics could surely clarify more details about the etiology and origin of this lesion [1].

A specific treatment is yet to be known [2]. Monotherapy with interferon $2 \alpha$ in one case showed significant improvements [2].

In conclusion, this case report emphasizes that these type of vascular lesion should be considered clinically and radiologically for prompt diagnostic procedures, immediate surgical intervention and control of bleeding. Also, surgical pathologists should always consider this lesion in differential diagnosis of vascular lesions of mediastinum.

\section{References}

1. Ammar A, EI Hammami S, Zaimi M, et al. An unusual lesion of the mediastinum: angiomatosis or diffuse hemangioma [abstract]. Ann Pathol 2004; 24: 54-7.

2. Eichler A, Ukena D, Wilkens $\mathrm{H}$, et al. Interferon alpha$2 \mathrm{a}$ in the treatment of diffuse thoracic angiomatosis in adulthood [abstract]. Pneumologie 2000; 54: 243-8.

3. Enzinger Weiss (eds). Soft tissue tumors. $4^{\text {th }}$. United states: Mosby, 2001; 873-5.

4. Zadoo VP, Leduc J. Mediastinal angiomatosis [abstract]. Mil Med 1995; 160: 362-3.

5. Hendrickx S, Hermans R, Wilms G, et al. Angiomatosis in the neck and mediastinum: an example of low flow vascular malformations [abstract]. Eur radiol 2003; 13: 981-5. 